\title{
Neutron Damage in
}

Mechanically-Cooled High-

Purity Germanium Detectors

for Field-Portable Prompt

Gamma Neutron Activation

Analysis (PGNAA) Systems

The INL is a

U.S. Department of Energy

National Laboratory

operated by

Battelle Energy Alliance

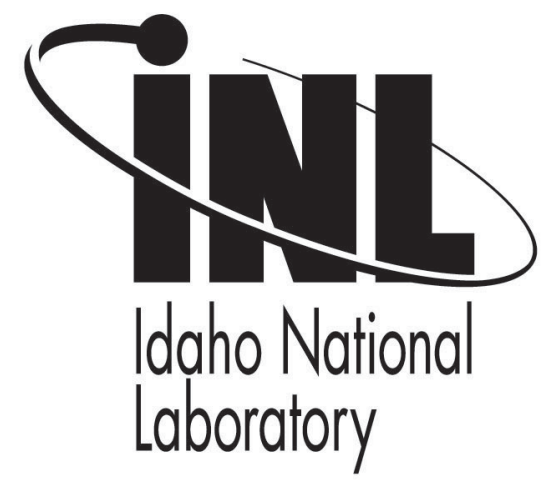

\section{IEEE Nuclear Science Symposium and Medical Imaging Conference}

E. H. Seabury

C. Dew. Van Siclen

J. B. McCabe

C. J. Wharton

A. J. Caffrey

\section{October 2013}

This is a preprint of a paper intended for publication in a journal or proceedings. Since changes may be made before publication, this preprint should not be cited or reproduced without permission of the author. This document was prepared as an account of work sponsored by an agency of the United States Government. Neither the United States Government nor any agency thereof, or any of their employees, makes any warranty, expressed or implied, or assumes any legal liability or responsibility for any third party's use, or the results of such use, of any information, apparatus, product or process disclosed in this report, or represents that its use by such third party would not infringe privately owned rights. The views expressed in this paper are not necessarily those of the United States Government or the sponsoring agency. 


\title{
Neutron Damage in Mechanically-Cooled High- Purity Germanium Detectors for Field-Portable Prompt Gamma Neutron Activation Analysis (PGNAA) Systems
}

\author{
E.H. Seabury, C. Dew. Van Siclen., J.B. McCabe, C.J. Wharton, and A.J. Caffrey Senior Member, IEEE
}

\begin{abstract}
Prompt Gamma Neutron Activation (PGNAA) systems require the use of a gamma-ray spectrometer to record the gamma-ray spectrum of an object under test and allow the determination of the object's composition. Field-portable systems, such as Idaho National Laboratory's PINS system, have used standard liquid-nitrogen-cooled high-purity germanium (HPGe) detectors to perform this function. These detectors have performed very well in the past, but the requirement of liquidnitrogen cooling limits their use to areas where liquid nitrogen is readily available or produced on-site. Also, having a relatively large volume of liquid nitrogen close to the detector can impact some assessments, possibly leading to a false detection of explosives or other nitrogen-containing chemical. Use of a mechanically-cooled HPGe detector is therefore very attractive for PGNAA applications where nitrogen detection is critical or where liquid-nitrogen logistics are problematic.

Mechanically-cooled HPGe detectors constructed from ptype germanium, such as ORTEC's TransSpec, have been commercially available for several years. In order to assess whether these detectors would be suitable for use in a fielded PGNAA system, Idaho National Laboratory (INL) has been performing a number of tests of the resistance of mechanicallycooled HPGe detectors to neutron damage. These detectors have been standard commercially-available $p$-type HPGe detectors as well as prototype $n$-type HPGe detectors. These tests compare the performance of these different detector types as a function of crystal temperature and incident neutron fluence on the crystal.
\end{abstract}

\section{INTRODUCTION}

TAHO National Laboratory's PINS system is a field-portable $\mathbf{I}_{\text {prompt gamma neutron activation analysis system that is }}$ currently used by the U.S. Army and others. The system is commercially available from the ORTEC division of Ametek [1]. As it is currently shipped, the system consists of a $40 \%$

Manuscript received November 15, 2013. This work was supported in part by the U.S. Army, Program Manager for Non-Stockpile Chemical Materiel. Idaho National Laboratory is operated by Battelle Energy Alliance for the U.S. Department of Energy, under DOE Field Office Idaho contract DE-AC07-05ID14517.

E.H. Seabury is with Idaho National Laboratory,Idaho Falls, ID, 83415 USA (telephone: 208-526-5303, e-mail edward.seabury@inl.gov).

C.DeW. Van Siclen, C.J. Wharton, and A.J. Caffrey are also with Idano National Laboratory.

J.B. McCabe is with the ORTEC division of Ametek, Oak Ridge, TN 37831 relative efficiency $n$-type high-purity germanium (HPGe) spectrometer, a $5 \mu \mathrm{g}{ }^{252} \mathrm{Cf}$ neutron source, notebook computer, and detector stand. A schematic of the system is shown in Figure 1 .

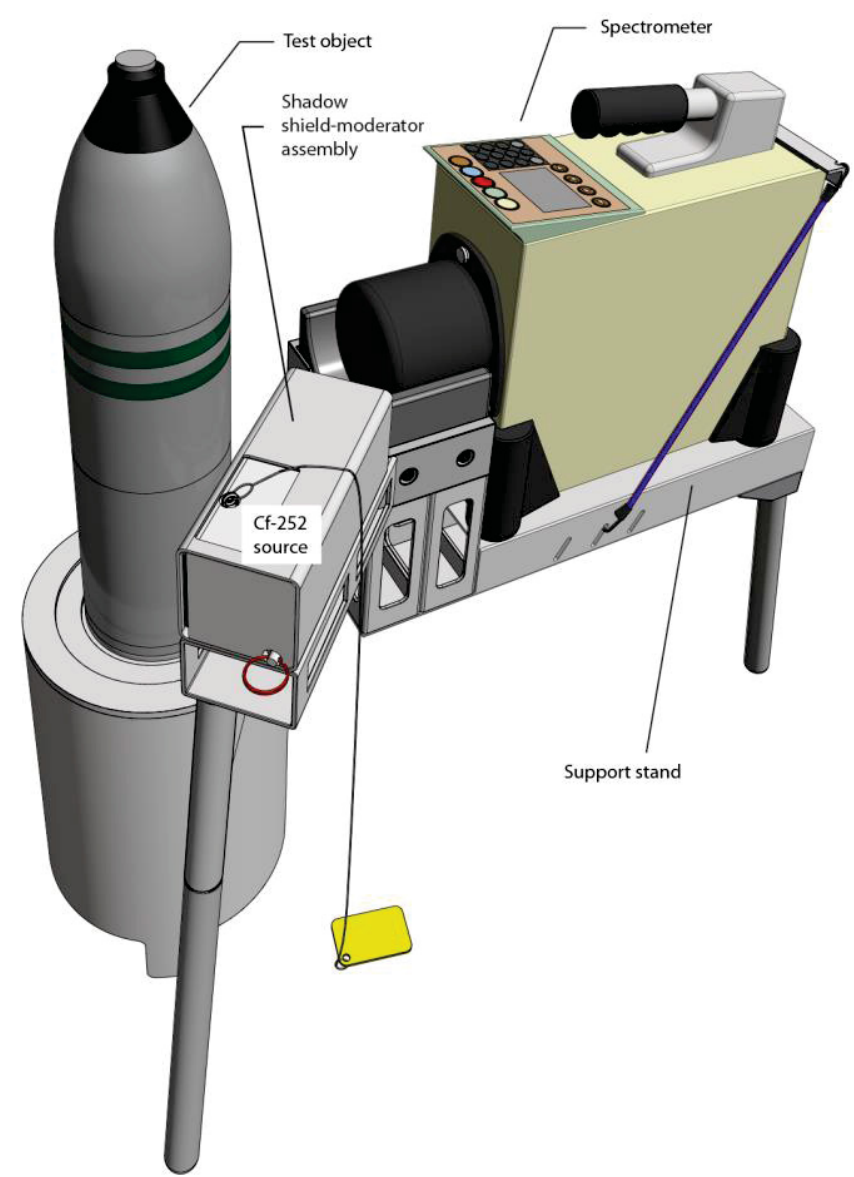

Fig. 1. INL's PINS system

The system is used to assess munitions [2] and determine their contents, i.e. whether they contain chemical warfare materiel (CWM), explosives, smoke-generating chemicals, or inert materials. Because the system is field-portable, it is generally used at the site where a munition has been recovered, 
and has been deployed by teams in Iraq and Afghanistan. These remote locations have made the logistics of obtaining liquid nitrogen problematic at times and there has therefore been significant interest in exchanging a mechanically-cooled HPGe detector for the standard LN2-cooled instruments. As part of this change we at INL have undertaken a study of the resistance of these mechanically-cooled HPGe detectors to neutron damage. There has been significant previous work in this area [3-4], but most recent studies have concentrated on detectors cooled with liquid nitrogen rather than at the higher temperatures generated by mechanical coolers.

Neutron damage in HPGe crystals becomes evident in the shape of peaks of the gamma-ray spectrum. An example of this can be found in Fig. 2 where the $1.33 \mathrm{MeV}$ peak from ${ }^{60} \mathrm{Co}$ can be seen both before and after neutron irradiation of a detector.

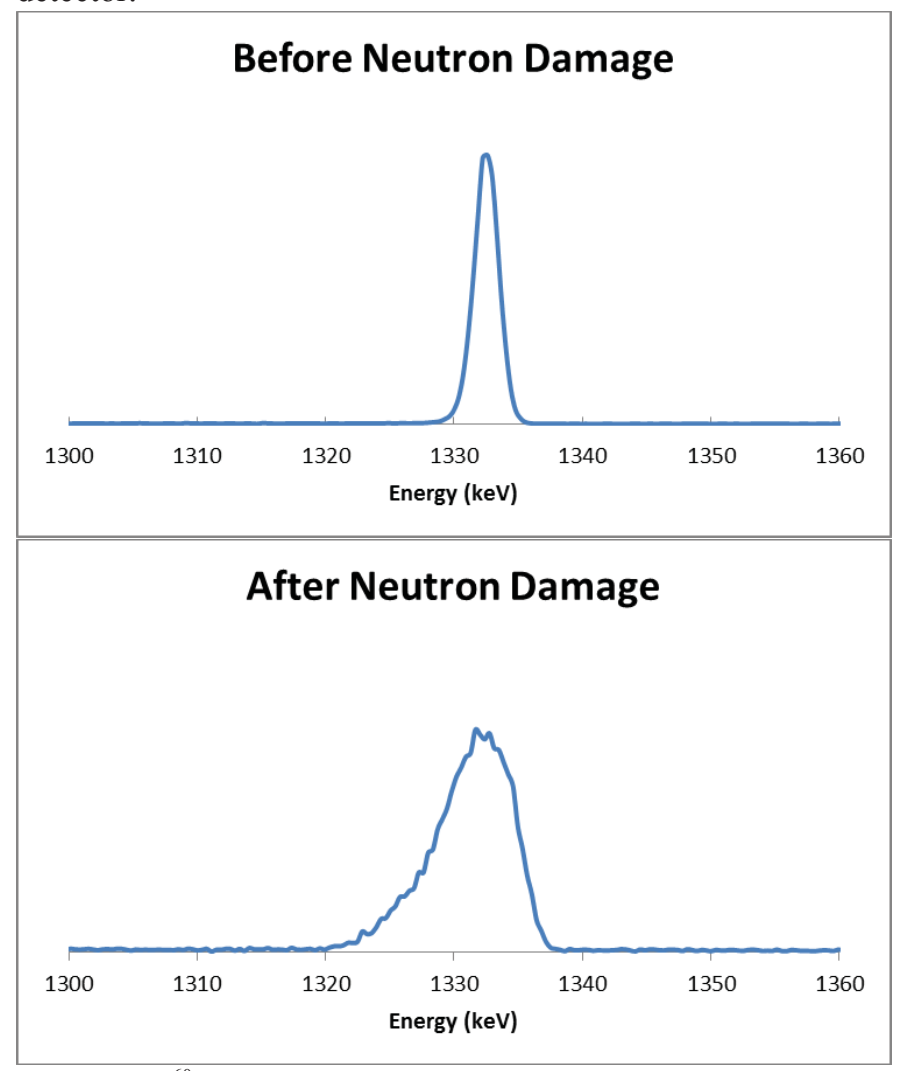

Fig. 2. ${ }^{60}$ Co peak before and after neutron damage of detector

This distortion of the peak shape is caused by chargecarriers in the HPGe crystal being trapped before they can reach the charge-collection electrodes. Fast neutrons can cause germanium atoms to be displaced from their usual positions in the crystal lattice, creating traps for chargecarriers and resulting in incomplete charge collection.

INL has conducted two separate experimental campaigns to examine the effects of neutron damage on mechanically-cooled detectors. The first was a series of irradiations on an ORTEC MicroDetective, where the effects of neutron damage were compared with those on a standard $n$-type LN2-cooled PINS detector. The results [5] of this experiment have been used to develop a phenomenological model of neutron damage and have been previously reported. We will concentrate here on the results of a second series of irradiations on the ORTEC TransSpec mechanically-cooled spectrometer.

The ORTEC TransSpec uses an approximately $40 \%$ relative efficiency HPGe crystal. These can be either of $n$ or $p$-type germanium. These measurements involved irradiations of both types of TransSpec.

\section{EXPERIMENTAL METHOD}

The experimental method for irradiating the detectors was very simple. A ${ }^{252} \mathrm{Cf}$ source emitting $\sim 1.1 \times 10^{7}$ neutrons/second was placed in a 4-inch by 4-inch polyethylene moderator block. This moderator block is the standard source holder in first-generations PINS systems and has a bismuth window in the front to attenuate low energy $\mathrm{x}$ and gamma rays leaving the source block. A steel plate was placed on the top of the moderator block to provide fiducial gamma rays from neutron interactions with iron. A photograph of the arrangement is shown in Fig. 3.

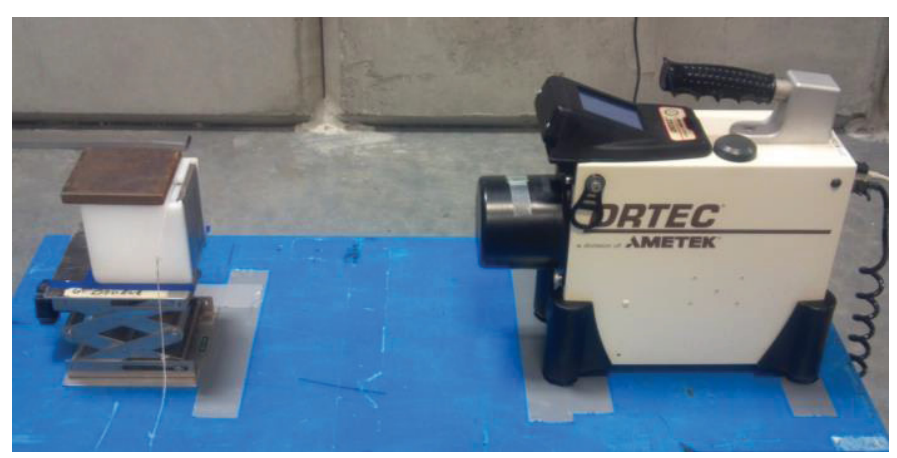

Fig. 3. Detector irradiation geometry

Neutrons were allowed to impinge on the front face of the detector. Gamma-ray spectra were measured during the irradiation, allowing monitoring of the performance of the detector. Periodically, the neutron source was removed and measurements of the energy resolution of the detector were made using a ${ }^{60} \mathrm{Co}$ source. The neutron irradiations were continued until the full width at half maximum (FWHM) of the $1.33 \mathrm{MeV}{ }^{60} \mathrm{Co}$ peak was measured to be approximately 6 $\mathrm{keV}$.

Four separate detector irradiations were carried out. These were irradiations of an $n$-type TransSpec with its cooler operating at $120 \mathrm{~K}$, an $n$-type TransSpec operating at $110 \mathrm{~K}$, a $p$-type TransSpec operating at $120 \mathrm{~K}$, and a $p$-type TransSpec operating at $110 \mathrm{~K}$.

\section{RESULTS}

Monte Carlo calculations of the neutron fluence on the face of the crystal were carried out using Los Alamos National Laboratory's MCNP code [6]. These calculations indicate that for a ${ }^{252} \mathrm{Cf}$ source emitting $1 \times 10^{7}$ neutrons/second, 1300 neutrons $/ \mathrm{cm}^{2} /$ second were impinging on the front face of the germanium crystal for each of the detectors. These 
calculations, combined with the measured activity of the ${ }^{252} \mathrm{Cf}$ source, were used to estimate the integrated neutron flux on each of the detector's germanium crystals.

\section{A. Cooler operating at $120 \mathrm{~K}$}

The first irradiations that were completed were those using the TransSpecs operating at $120 \mathrm{~K}$. Plots of the full width at tenth maximum (FWTM) and FWHM of the ${ }^{60} \mathrm{Co} 1.33 \mathrm{MeV}$ peak for both the $n$-type and $p$-type detectors can be seen in Figs. 4 and 5. These figures show the expected [7] degradation in energy resolution as the integrated neutron flux on the detectors increases. They also show the expected resistance to neutron damage of the $n$-type detector when compared to the $p$-type.

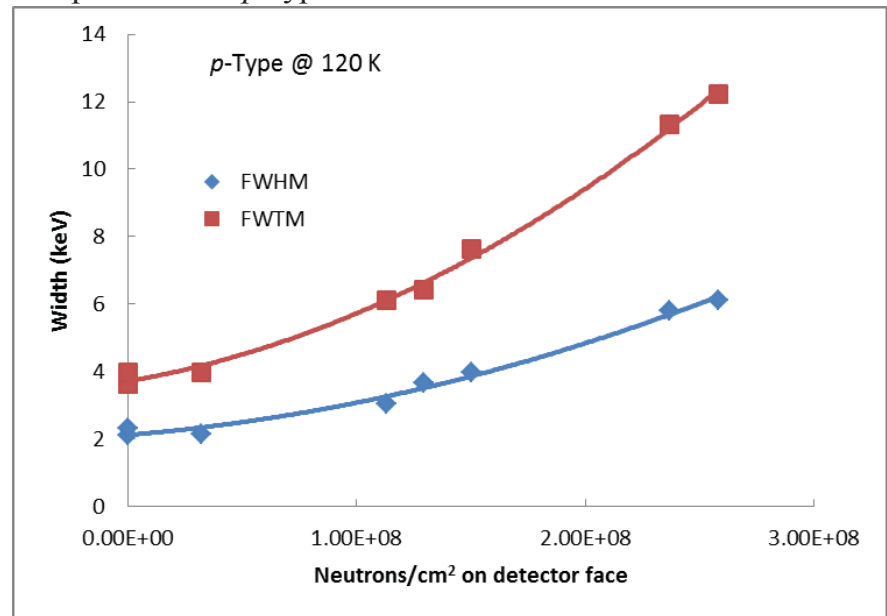

Fig. 4. FWHM and FWTM for $p$-type TransSpec operating at $120 \mathrm{~K}$

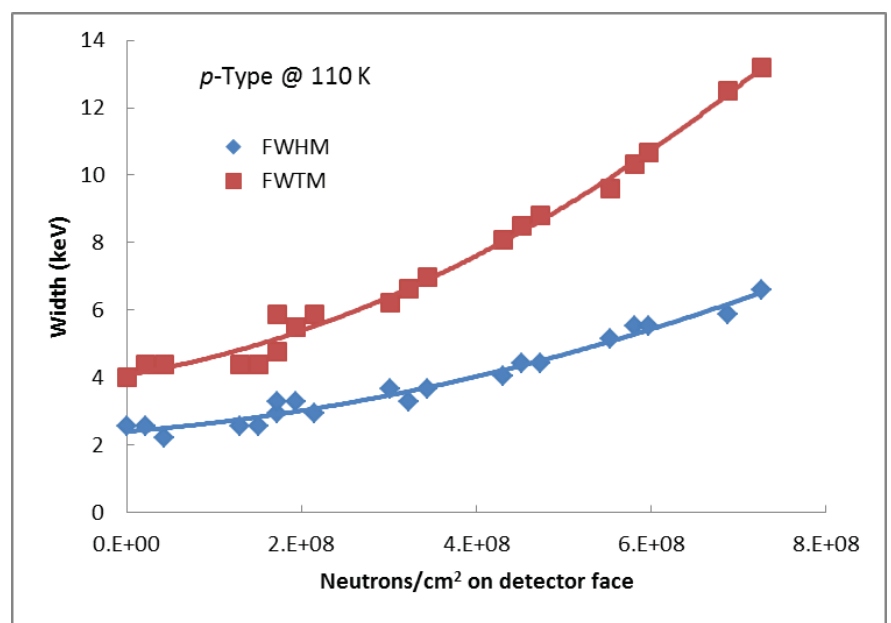

Fig. 6. FWHM and FWTM for $p$-type TransSpec operating at $120 \mathrm{~K}$

The two Figs also show the more rapid degradation in the FWTM when compared with the FWHM. This degradation of the FWTM is particularly significant for PINS measurements given that the spectra produced by neutron interrogation of a munition have many strong peaks, in particular from neutron interactions with the steel body of the munition which comprises most of its mass. Peaks produced by explosives or CWM within the munition are frequently weak when compared with those produced by the body of the munition and can therefore be more difficult to identify and fit when merged with the low-energy tail caused by neutron damage of the germanium crystal.

\section{B. Cooler operating at $110 \mathrm{~K}$}

The results for the $n$ and $p$-type TransSpecs with the cooler operating at $110 \mathrm{~K}$ can be seen in Figs 6 and 7. These plots show that there is significantly more resistance to neutron damage for both of these detectors when compared with the results with the cooler operating at $120 \mathrm{~K}$.

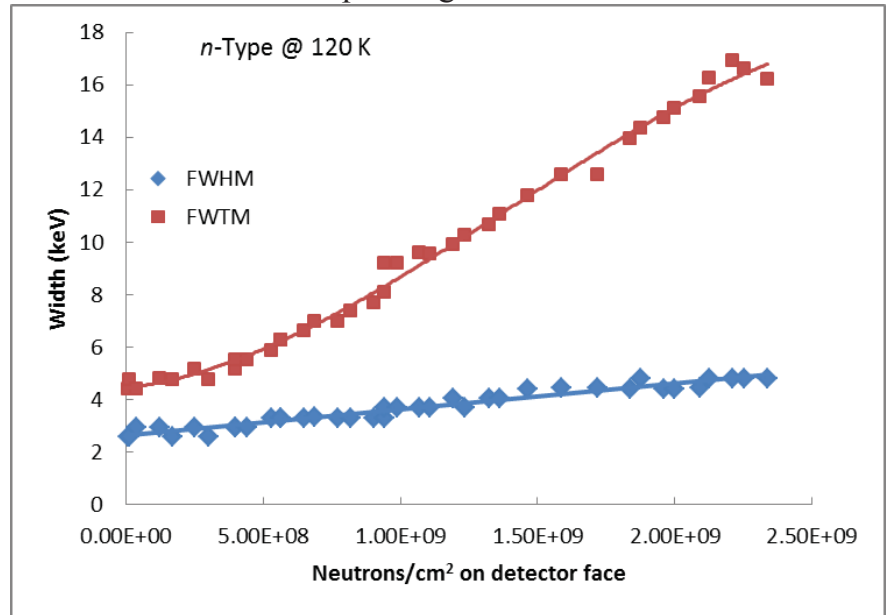

Fig. 5 FWHM and FWTM for $n$-type TransSpec operating at $110 \mathrm{~K}$

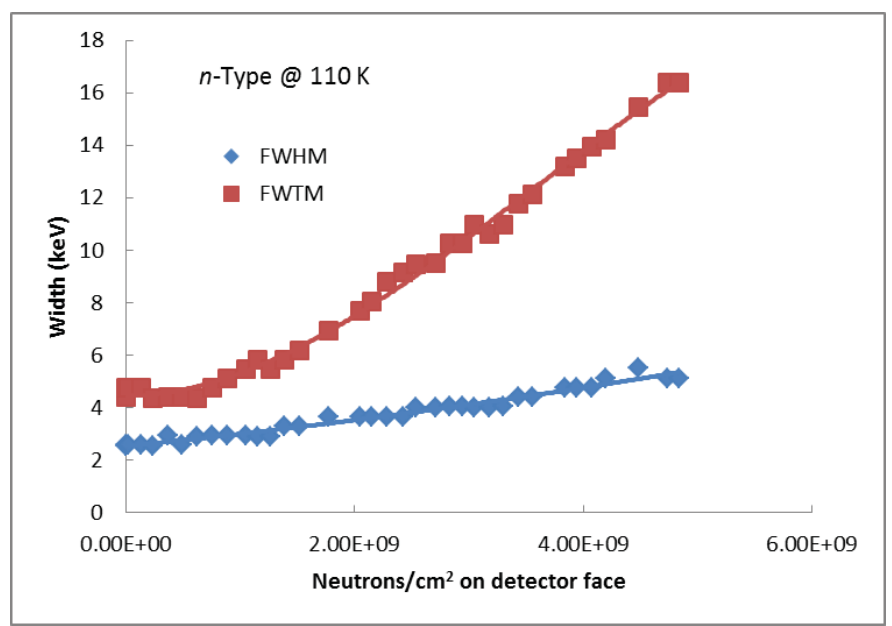

Fig. 7. FWHM and FWTM for $n$-type TransSpec operating at $110 \mathrm{~K}$

\section{CONCLUSIONS}

The use of $p$-type TransSpecs appears to be severely limited by the neutron damage they can sustain in a fielded active interrogation system. This applies in particular to those operated at $120 \mathrm{~K}$ where irradiations on the order of 60 hours made the systems unable to perform PINS assessments of munitions. The neutron flux on the detector is somewhat less in a standard PINS measurement and MCNP calculations of the flux in this geometry indicate that the detector could be operated for approximately 90 hours before being too degraded for additional use. 
Changes in the operating temperature of the mechanical cooler showed significant improvement in the resistance of the detectors to neutron damage, showing approximately a factor of two improvement by lowering the operating temperature to $110 \mathrm{~K}$ from $120 \mathrm{~K}$. This effectively doubles the lifetime of a detector before it is no longer suitable for use. This drastic improvement in the resistance to neutron damage warrants further investigation, in particular the effects of an even lower operating temperature on both the resistance to neutron damage and the initial energy resolution of the detector.

\section{ACKNOWLEDGMENT}

The authors would like to thank their INL colleagues Ann Egger and Ken Krebs, and their ORTEC colleague K.C. Thompson for their support of these measurements.

\section{REFERENCES}

[1] ORTEC Website http://www.ortec-online.com

[2] A.J. Caffrey et al., "Chemical Warfare Agent and High Explosive Identification by Spectroscopy of Neutron-Induced Gamma Rays," IEEE Trans. Nucl. Sci., vol. 39, no. 5, pp. 1422-1426, Oct. 1992.

[3] L.S. Darken, "Role of disordered regions in fast-neutron damage of HPGe detectors", Nuc. Instr. and Meth., B74, 1993 (523-526).

[4] M. Descovich et al., "Effects of neutron damage on the performance of large volume segmented germanium detectors", Nuc. Instr. And Meth. A, 545, 2005, pp 199-209.

[5] C.Dew. Van Siclen et al., "Phenomenological Model for Predicting the Energy Resolution of Neutron-Damaged High-Purity Germanium Detectors," IEEE Trans. Nucl. Sci., vol. 59, no. 5, pp. 2487-2493, Oct. 2012.

[6] X-5 Monte Carlo Team, "MCNP A General Monte Carlo N-Particle Transport Code, Version 5," Los Alamos Report LA-UR-03-1987, Feb. 2008.

[7] Glen Knoll, Radiation Detection and Measurement $2^{\text {nd }}$ Edition, John Wiley \& Sons, New York, 1989, pp 409-440. 